\title{
1 In situ monitoring of powder blending by non-invasive Raman 2 spectrometry with wide area illumination
}

3 Pamela Allan, ${ }^{a}$ Luke J. Bellamy, ${ }^{\mathrm{a}, 1}$ Alison Nordon, ${ }^{\mathrm{a} *}$ David Littlejohn, ${ }^{\mathrm{a} *}$ John Andrews ${ }^{\mathrm{b}}$

4 and Paul Dallin

$5{ }^{a}$ WestCHEM, Department of Pure and Applied Chemistry and CPACT, University of

6 Strathclyde, 295 Cathedral Street, Glasgow, G1 1XL, UK

7 b Clairet Scientific Ltd., 17/18 Scirocco Close, Moulton Park Industrial Estate,

8 Northampton, NN3 6AP, UK

$9 \quad{ }^{1}$ Present address: GlaxoSmithKline, Priory Street, Ware, SG12 0DJ, UK.

10

11 * denotes authors to whom correspondence should be sent

12 (Email: d.littlejohn@strath.ac.uk and alison.nordon@strath.ac.uk)

\section{Abstract}

15 A $785 \mathrm{~nm}$ diode laser and probe with a $6 \mathrm{~mm}$ spot size were used to obtain spectra of

16 stationary powders and powders mixing at $50 \mathrm{rpm}$ in a high shear convective blender.

17 Two methods of assessing the effect of particle characteristics on the Raman sampling

18 depth for microcrystalline cellulose (Avicel), aspirin or sodium nitrate were compared: (i)

19 the information depth, based on the diminishing Raman signal of $\mathrm{TiO}_{2}$ in a reference

20 plate as the depth of powder prior to the plate was increased, and (ii) the depth at which a

21 sample became infinitely thick, based on the depth of powder at which the Raman signal 
22 of the compound became constant. The particle size, shape, density and/or light

23 absorption capability of the compounds were shown to affect the "information" and

24 "infinitely thick" depths of individual compounds. However, when different sized

25 fractions of aspirin were added to Avicel as the main component, the depth values of

26 aspirin were the same and matched that of the Avicel: $1.7 \mathrm{~mm}$ for the "information"

27 depth and $3.5 \mathrm{~mm}$ for the "infinitely thick" depth. This latter value was considered to be

28 the minimum Raman sampling depth when monitoring the addition of aspirin to Avicel in

29 the blender. Mixing profiles for aspirin were obtained non-invasively through the glass

30 wall of the vessel and could be used to assess how the aspirin blended into the main

31 component, identify the end point of the mixing process (which varied with the particle

32 size of the aspirin), and determine the concentration of aspirin in real time. The Raman

33 procedure was compared to two other non-invasive monitoring techniques, near infrared

34 (NIR) spectrometry and broadband acoustic emission spectrometry. The features of the

35 mixing profiles generated by the three techniques were similar for addition of aspirin to

36 Avicel. Although Raman was less sensitive than NIR spectrometry, Raman allowed

37 compound specific mixing profiles to be generated by studying the mixing behaviour of

38 an aspirin - aspartame - Avicel mixture.

$39<$ Take in Figure 1>

$40 \quad$ Keywords

41 Raman spectrometry; process analytical technologies (PAT); powder blending; sampling

42 depth; real-time monitoring; pharmaceuticals. 


\section{$44 \quad$ 1. Introduction}

45 Raman spectrometry is proving to be a useful monitoring technique in the pharmaceutical

46 industry, especially in secondary manufacturing $[1,2]$. Considerable advantages have

47 been demonstrated for analysis of tablets [3-18] and capsules [11, 19-23], particularly

48 when transmission mode measurements were used [16-18, 20-24]. To ensure that

49 pharmaceutical dosage forms contain the appropriate amount of active ingredient(s), the

50 constituents must be blended to a homogeneous state. While transmission Raman

51 spectrometry is suited for the analysis of tablets and capsules, the backscatter mode of

52 measurement is more amenable for in situ analysis of larger unit operations such as

53 powder blending. However, there are relatively few reports describing the use of

54 backscatter Raman spectrometry for this purpose [25-27]; in contrast, use of in situ near

55 infrared (NIR) spectrometry is far more common [28-33]. The mixing of diltiazem

56 hydrochloride pellets and paraffinic wax was investigated by Vergote et al. using non-

57 invasive Raman spectrometry [26]. The process was monitored via a glass window in the

58 side of the vessel using a laser spot of approximately $2 \mathrm{~mm}$ diameter. There was no

59 significant difference in the intensity of the Raman signal when the diltiazem pellets were

60 stationary or mixing at $50 \mathrm{rpm}$. Therefore, spectra could be recorded without stopping the

61 mixing procedure and the Raman signal became constant when homogeneity had been

62 reached. De Beer et al. [25] monitored the blending of Avicel PH 102, lactose DCL 21,

63 dilitazem hydrochloride, and silicium dioxide using an invasive Raman probe; the end of

64 the probe was flush with the inner surface of the mixing vessel wall. The mixing end-

65 point identified from the Raman measurements was comparable to that obtained using

66 NIR spectrometry. Hausman et al. also reported the successful implementation of in-line 
67 Raman spectrometry to monitor blending of azimilide dihydrochloride at low dose $68(1 \% \mathrm{w} / \mathrm{w})[27]$.

69 One reason for the limited application of Raman spectrometry to powder 70 processes is that conventional backscatter Raman systems typically employ optics that 71 produce laser spot sizes smaller than $500 \mu \mathrm{m}$ diameter, which results in the measurement 72 of only a small volume of the sample. More representative sampling has been achieved 73 by continuously rotating a sample during measurement $[5,6,9,10,13,14,34]$ or by 74 scanning at several positions $[8,34,35]$, thereby increasing the sampling area. To 75 overcome some of the sub-sampling limitations of conventional backscatter Raman 76 systems, defocused probes or wide area sampling optics with laser beam diameters of 3 $777 \mathrm{~mm}$ have also been investigated [15, 36-38]. Backscatter Raman measurements also 78 exhibit a strong bias towards the upper surface layers of the sample. Monte Carlo 79 simulations of the backscattered Raman signal from a $4 \mathrm{~mm}$ thick tablet using a $4 \mathrm{~mm}$ 80 diameter laser spot have shown that $88 \%$ of the signal is generated in the top $1 \mathrm{~mm}$ layer 81 of the tablet [24]. However, the increased sampling depth and area of wide area 82 illumination probes results in significantly larger sampling volumes compared to

83 conventional backscatter Raman probes. For example, it has been shown that Raman 84 signals can originate from material $13 \mathrm{~mm}$ below the surface of a sample with a PhAT 85 probe with a $7 \mathrm{~mm}$ spot size [38]. The sampling volume of a PhAT probe (3 mm spot 86 size) was found to be approximately 1300 times larger than that of a non-contact Raman 87 probe $(150 \mu \mathrm{m}$ spot size $)$ and approximately 16700 times larger than that of an 88 immersion probe (60 $\mu \mathrm{m}$ spot size) [36]. The larger sampling volume associated with the

89 PhAT probe resulted in comparable results to NIR spectrometry for quantification of 
90 mixtures of anhydrous and hydrated polymorphs; the sampling volume of the PhAT

91 probe was found to be comparable to that for the NIR probe [39]. When mixtures of

92 polymorphs of flufenamic acid (forms I and III) with different particle sizes were

93 analysed using large and small spot size Raman probes, the wide area illumination probe

94 was found to be less sensitive to particle size owing to the larger sampling volume

95 measured with this probe [37].

96 This report describes the first use of a PhAT probe for in situ Raman monitoring

97 of powder mixing in a high shear blender. The study included evaluation of the effect of

98 particle size variations on the sampling depth that can be achieved with the PhAT probe

99 to allow comparison with previously reported results obtained with probes of different

100 optical configurations. In addition, the effect of particle size on the Raman signal was

101 investigated; this was important as the effect of particle size on the backscatter Raman

102 signal is highly dependent on the optical configuration of the probe employed [40].

103 Mixing profiles based on non-invasive Raman spectrometry have been assessed to

104 compare the information they provide with mixing profiles produced by non-invasive

105 NIR spectrometry and broadband acoustic emission (AE) spectrometry. Some advantages

106 of Raman measurements over NIR spectrometry were identified for powder monitoring. 107 


\section{2. Experimental}

\section{$109 \quad$ 2.1. Instrumentation}

\section{$110 \quad$ 2.1.1. Raman spectrometer}

111 A Kaiser Raman RXN1 spectrometer with a PhAT probe (Kaiser Optical Systems Inc.,

112 Ann Arbor. MI, USA) was used for all experiments except where stated below. The

$113785 \mathrm{~nm}$ Invictus diode laser was operated at $400 \mathrm{~mW}$ at source. The laser beam was

114 optically expanded to give a $6 \mathrm{~mm}$ spot size, a working distance of $254 \mathrm{~mm}$ and a depth

115 of field of $50 \mathrm{~mm}$. The probe to sample distance was $203 \mathrm{~mm}$. For the sampling depth

116 studies, spectra were acquired using an exposure time of $0.5 \mathrm{~s}$ and 1 accumulation. In situ

117 Raman measurements were made through the glass wall of the mixing vessel during the

118 powder blending experiments. A spectrum was acquired every $5 \mathrm{~s}$ with an exposure time

119 of $2.5 \mathrm{~s}$ and 1 accumulation. The exposure time for each set of experiments was selected

120 such that the largest Raman signal occupied approximately $60-70 \%$ of the dynamic

121 range of the CCD detector.

122 For the experiment comparing Raman, NIR and AE, and the monitoring of the

123 blending of a three component mixture, a different PhAT probe system was used. In this

124 case, the $785 \mathrm{~nm}$ laser beam was optically expanded to give a $3 \mathrm{~mm}$ spot size and a

125 working distance of $100 \mathrm{~mm}$. The probe to sample distance was $100 \mathrm{~mm}$. Spectra were 126 acquired every $3 \mathrm{~s}$, through the wall of the vessel, with an exposure time of $2 \mathrm{~s}$ and 1 127 accumulation.

128 A spectrum of aspartame was acquired using a Kaiser RXN1 spectrometer with a

129 MR probe equipped with a non-contact optic ( 0.4 inch working distance and a laser spot 
130 size of approximately $100 \mu \mathrm{m})$. An Invictus diode laser with a wavelength of $785 \mathrm{~nm}$ was

131 employed, and was operated at $350 \mathrm{~mW}$ at the source. Aspartame was contained within a

132 glass sample vial, which was placed in the off-line sample compartment, and the

133 spectrum was acquired through the side of the vial using an exposure time of $4.0 \mathrm{~s}$ and 1

134 accumulation.

135 Data were acquired using HoloGRAMS software (Kaiser Optical Systems). A

136 dark current spectrum was obtained before each set of experiments and subtracted

137 automatically from each subsequent spectrum acquired. Data were exported into

138 GRAMS/32 and converted to text files, which were subsequently imported into Matlab

1397.0 (Mathworks Inc., Natick, Massachusetts, USA) for analysis using the PLS_Toolbox

140 version 3.04 (Eigenvector Research Inc., Manson, Washington, USA). As spectra of

141 samples containing Avicel exhibited a sloping baseline arising from fluorescence, second

142 derivative spectra were calculated throughout using the Savitzky - Golay function with a

143 second order polynomial and a 13 or 25 point filter width for data acquired using the 6 or

$1443 \mathrm{~mm}$ laser spot size, respectively.

\section{2.1.2. Near infrared reflectance spectrometer}

146 Some measurements of powder mixing were obtained simultaneously by non-invasive

147 Raman spectrometry, NIR spectrometry and broadband AE spectrometry. In situ NIR 148 measurements were made through the glass wall of the vessel as described previously 149 [31, 32] using a Zeiss Corona 45 NIR spectrometer (Carl Zeiss, Heidenheim, Germany).

150 Measurements were acquired using Aspect software (Carl Zeiss) package and spectra

151 stored as $\log (1 / \mathrm{R})$ where $\mathrm{R}$ is the reflectance and was calculated from the intensity of 152 light reflected by the sample relative to that for a reflectance standard. The integration 
153 time was $32 \mathrm{~ms}$ and 10 scans were co-added for each acquired spectrum allowing

154 measurements to be taken every $0.5 \mathrm{~s}$. Data were exported as text files into Matlab for

155 further analysis using PLS_Toolbox. First derivative spectra were calculated using the

156 Savitzky - Golay function with a 5 point filter width and second order polynomial.

\section{2.1.3. Acoustic emission}

158 The broadband AE monitoring equipment has been described previously [41]. A Nano 30

159 transducer (Physical Acoustics Ltd, Cambridge, UK) was attached to the glass wall of the

160 mixing vessel using a silicone-based vacuum grease (Dow Corning) and adhesive tape.

161 The Nano 30 transducer was attached to a 2/4/6 series pre-amplifier (Physical Acoustics

162 Limited). The pre-amplifier required a $28 \mathrm{~V}$ power supply (Physical Acoustics Limited)

163 and the gain of the pre-amplifier was set to $60 \mathrm{~dB}$. The pre-amplifier was connected to an

164 Agilent 54642A oscilloscope using a $5 \mathrm{~m}$ length cable, which was linked to a computer via a

165 GPIB to USB interface (Agilent Technologies).

166 A data capture program, written in $\mathrm{C}^{++}$by Douglas McNab and Robbie Robinson

167 from the Centre of Ultrasonic Engineering (CUE) at the University of Strathclyde,

168 enabled the cyclic sampling of the acoustic signals displayed on the oscilloscope and

169 signals were saved as comma separated variable (CSV) files. Signals were acquired using

170 a sampling rate of $2 \mathrm{MHz}$ and the time interval between collection of signals was $2 \mathrm{~s}$. A

171 total of 450 signals were collected with each signal consisting of 4000 points. All

172 acoustic signals were imported into Matlab for analysis. A power spectrum was

173 calculated of each signal and three spectra were co-added to give a composite spectrum

174 every 6 s. Signal areas were calculated by summing the intensities of the signals between

1750 and $400 \mathrm{kHz}$. 


\section{2.2. Powders}

178 Microcrystalline cellulose (Avicel PH-101; FMC, Cork, Ireland), aspirin, sodium nitrate

179 (both from Sigma-Aldrich, Dorset, UK) and aspartame (provided by GSK, UK) were

180 used. The Avicel particles have a tap density of $0.45 \mathrm{~g} \mathrm{~cm}^{-3}$, are granular in shape and

181 have an average particle size of $50 \mu \mathrm{m}$. Aspirin particles are low aspect ratio needles with

182 an average particle size of $192 \mu \mathrm{m}$. Sodium nitrate particles are granular with an average

183 particle size of $275 \mu \mathrm{m}$. Aspartame particles are high aspect needles (particle size was not

184 measured). Average particle size information was obtained by laser diffraction, while

185 density and shape information was obtained from the literature [42]. The powders were

186 sieved through $10 \mathrm{~cm}$ diameter brass pan sieves (Endecotts Ltd, UK) to obtain different

187 particle size ranges: $<38,38-53,53-106,150-212,212-250,250-300,300-355$

188 and $355-425 \mu \mathrm{m}$ for Avicel; <106, $106-150,212-250,250-300,300-355$, and 425

$189-500 \mu \mathrm{m}$ for aspirin; $150-212,212-250,250-300,300-355,355-425,425-500$

190 and $500-800 \mu \mathrm{m}$ for sodium nitrate. The mid-point of the sieve range has been used in

191 plots to represent each particle size fraction.

\section{2.3. Procedures}

\section{$194 \quad$ 2.3.1. Raman sampling depth studies}

195 A $2.68 \mathrm{~mm}$ thick layer of glass was placed on top of the inverted PhAT probe with a $196203 \mathrm{~mm}$ spacer attached (Figure 1a). A series of plastic plates, with a $30 \mathrm{~mm}$ diameter 197 hole, were placed on top of the glass plate (Figures $1 \mathrm{~b}$ and 1c). For investigation of 
198 powder depths of $0-4.48 \mathrm{~mm}$, up to $16 \times 0.28 \mathrm{~mm}$ thick plates were stacked on top of

199 each other. A depth of $8.48 \mathrm{~mm}$ was obtained by placing an additional $4 \mathrm{~mm}$ thick plastic

200 plate on top of the 16 plates. This combination of plates enabled sampling depths of

$201 \leq 4.48 \mathrm{~mm}$ to be measured. The powder was carefully placed into the hole in the plastic

202 plates and levelled off using a razor blade, ensuring minimal compaction of the solid, and

203 the mass of solid was recorded. $\mathrm{A} \mathrm{TiO}_{2}$ reference layer (solid particles of $\mathrm{TiO}_{2}$ sealed

204 between two microscope slides) was placed over the top layer of the powder (Figures 1b

205 and 1c) with aluminium foil used as the final layer to reduce loss of laser and Raman

206 photons from the upper surface of the $\mathrm{TiO}_{2}$ reference layer. Each corner of the

207 microscope slide was labelled to ensure consistency in positioning, so that the same part

208 of the $\mathrm{TiO}_{2}$ was analysed each time. A spirit level was placed on top of the glass above

209 the spectrometer to ensure the radiation was pointing vertically upwards (see Figure 1a).

210 Analysis of the variance of the Avicel signal at $1095 \mathrm{~cm}^{-1}$ revealed that the repeatability

211 (expressed as the relative standard deviation (RSD) for $n=6$ ) of the Raman

212 measurements, positioning of the sample holder, the weighing procedure and the powder

213 levelling process were $0.22,0.34,0.66$, and $0.26 \%$, respectively.

214 The information depth was estimated using the $\mathrm{TiO}_{2}$ Raman signal at $397 \mathrm{~cm}^{-1}$. As

215 the depth of Avicel, aspirin or sodium nitrate increased, the $\mathrm{TiO}_{2}$ signal decreased until it

216 became zero; hence, the information depth was defined as the point where the exciting

217 laser can no longer penetrate the powder to generate a detectable Raman signal of the

$218 \mathrm{TiO}_{2}$ reference layer. This type of approach has been used previously for information

219 depth measurements in NIR reflectance spectrometry [31, 43, 44]. A second set of

220 measurements was made based on the change in the intensity of the Raman signals of 
221 Avicel, aspirin and sodium nitrate powders at 1095,1606 and $1068 \mathrm{~cm}^{-1}$, respectively. In

222 this case, the Raman signal increases with powder depth and then becomes constant,

223 which defines the depth of powder at which the sample effectively becomes infinitely

224 thick. These measurements were made to allow comparisons with the results of previous

225 Raman studies [36, 40] and a study on reflectance NIR which used a similar protocol to

226 assess the sampling depth for aspirin powders [31].

\section{2.3.2. Powder blending}

228 The scaled-down convective mixer has been described previously [31, 32, 41]. The vessel

229 has a pot size of about $500 \mathrm{~mL}$, a radius of $4 \mathrm{~cm}$ and is made of glass. The impeller has

230 three blades set $120^{\circ}$ apart with a tilt angle of $45^{\circ}$; each blade is approximately $29 \mathrm{~mm}$

231 long and $12 \mathrm{~mm}$ wide. Powders were mixed at $50 \mathrm{rpm}$ using a stirrer motor (IKA

232 Eurostar, VWR International).

233 For the mixing of two components, $75 \mathrm{~g}$ of unsieved Avicel PH-101 was placed

234 into the vessel and mixed. After $120 \mathrm{~s}$, different masses $(0,5,10,20,30$ or $40 \mathrm{~g})$ of either

235 unsieved or size fractions of sieved aspirin $(<106,250-300$ or $425-500 \mu \mathrm{m})$ were

236 added via a funnel positioned directly above the centre of the vessel and the powders

237 were allowed to mix for a further $780 \mathrm{~s}$. A further experiment was also conducted in

238 which $25 \mathrm{~g}$ of unsieved aspirin and $25 \mathrm{~g}$ of unsieved aspartame was added to $75 \mathrm{~g}$ of

239 unsieved Avicel PH-101 after 120 and 900 s, respectively; the total mixing time was

$2402100 \mathrm{~s}$. 


\section{Results and Discussion}

\section{3.1. Raman spectra}

244 Individual Raman spectra of aspirin, Avicel, and sodium nitrate measured through a glass

245 plate, aspartame measured through the wall of a glass vial, the $\mathrm{TiO}_{2}$ reference layer, and

246 the glass plate are illustrated in Figure 2. The Raman spectrum of $\mathrm{TiO}_{2}$ contains peaks at

247 approximately $143,397,520$ and $640 \mathrm{~cm}^{-1}$, indicating that the $\mathrm{TiO}_{2}$ is predominantly in

248 the anatase phase [45]. The broad peak that appears at approximately $1400 \mathrm{~cm}^{-1}$ in all

249 spectra in Figure 2 can be attributed to the glass plate.

$250 \quad \mathrm{The}^{\mathrm{TiO}}$ peak at $397 \mathrm{~cm}^{-1}$ was least affected by the other compounds and so was

251 used for the information depth experiments. The peaks at 1068 and $1606 \mathrm{~cm}^{-1}$ in the

252 sodium nitrate and aspirin spectra, respectively, were used to determine the depth at

253 which the sample becomes infinitely thick. A small peak in the Avicel spectrum at

254 approximately $1095 \mathrm{~cm}^{-1}$ was also measured, but was found to have poor sensitivity

255 compared to the peaks selected for the other two compounds.

\section{3.2. Raman information depths for uncompacted powders}

258 A number of particle size ranges of Avicel, aspirin and sodium nitrate were analysed at

259 increasing depths using the set-up illustrated in Figure 1, and the second derivative

260 Raman spectral intensities were recorded. When the $\mathrm{TiO}_{2}$ peak at $397 \mathrm{~cm}^{-1}$ could no

261 longer be detected, the depth of the selected powder was deemed to be the information

262 depth limit. Figure 3 gives a summary of the information depths for the different particle

263 size fractions of each compound. In each case, the information depth increased with 
264 particle size before becoming constant at higher particle sizes. As the information depth

265 for $300-355 \mu \mathrm{m}$ aspirin and $355-425 \mu \mathrm{m}$ sodium nitrate particles was found to be

$266>4.48 \mathrm{~mm}$ and so could not be determined, values for these fractions were not included in

267 Figure 3. The increase in information depth with particle size is consistent with Kubelka-

268 Munk theory. Diffuse reflectance decreases as particle size increases [46, 47] and

269 consequently, the exciting laser intensity and the Raman signal generated can propagate

270 through larger depths of powder. The differences in information depth for similar sizes of

271 Avicel, aspirin and sodium nitrate particles $<400 \mu \mathrm{m}$ indicates that the particle shape,

272 density and/or the light absorption capability of the compounds affect the depth of

273 powder through which the $\mathrm{TiO}_{2}$ Raman spectrum can be measured.

274 As the intention was to use the PhAT probe to monitor powder blending in a 275 mixer, information depths for $\mathrm{TiO}_{2}$ at $397 \mathrm{~cm}^{-1}$ were obtained for 10,30 and $40 \mathrm{~g}$ of 276 aspirin blended with $75 \mathrm{~g}$ Avicel PH-101 to give aspirin concentrations of 11.8, 28.6 and $27734.8 \% \mathrm{w} / \mathrm{w}$, respectively. Three particle size ranges of aspirin were used: $<106,250-$ 278300 and $425-500 \mu \mathrm{m}$. The information depth for each of the mixtures was in the range $279 \quad 1.7-2.0 \mathrm{~mm}$, similar to the value for unsieved Avicel PH-101 $(1.7 \mathrm{~mm})$. These findings 280 are similar to those obtained using NIR spectrometry, i.e. the information depth for the 281 analyte compound is determined by the main component in the mixture [31].

\section{3.3. Depth of powder at which sample becomes infinitely thick}

284 The depth of powder at which the $2^{\text {nd }}$ derivative Raman signal of Avicel $\left(1095 \mathrm{~cm}^{-1}\right)$, 285 aspirin $\left(1606 \mathrm{~cm}^{-1}\right)$ or sodium nitrate $\left(1068 \mathrm{~cm}^{-1}\right)$ became constant was different for each

286 compound and varied with particle size. The depth was found to be $>3 \mathrm{~mm}$ for each 
287 particle size range for Avicel. For sodium nitrate, the depth for the two smallest fractions $288(150-212 \mu \mathrm{m}$ and $212-250 \mu \mathrm{m})$ was about $0.8-1 \mathrm{~mm}$, whereas for the other particle 289 sizes, the value was $3.5-3.9 \mathrm{~mm}$. For aspirin, the depth initially increased with 290 increasing particle size (from 3.8 to $4.5 \mathrm{~mm}$ ) then decreased to $3.5 \mathrm{~mm}$ for the largest 291 aspirin particle size range $(425-500 \mu \mathrm{m})$ analysed. When other peaks in the aspirin 292 spectrum $\left(292,751\right.$ and $\left.1045 \mathrm{~cm}^{-1}\right)$ were measured, the information depth values were no 293 different to those obtained at $1606 \mathrm{~cm}^{-1}$. In contrast, it has been shown that different 294 peaks in the NIR spectrum of aspirin give different "infinitely thick" sample depths 295 owing to the different absorptivities of the first and second overtones in the spectrum (0.6 $296-1.1 \mathrm{~mm}$ and $1.1-2.2 \mathrm{~mm}$, respectively, depending on particle size) [31].

297 Wang et al. [40] investigated the effect of particle characteristics on the Raman 298 sampling depth for a number of crystalline powders using the "infinitely thick" method 299 and found similar trends to those reported here. An increase in sampling depth was 300 observed with average particle size in the range $108-428 \mu \mathrm{m}$ for sodium nitrate; 301 however, the values obtained $(6-15.5 \mathrm{~mm})$ were much larger than those obtained in the 302 present study. This is likely to be due to a combination of factors; the laser wavelength $303(514.5 \mathrm{~nm})$ was shorter (sampling depth is wavelength dependent [48]) and a different 304 optical configuration was employed, which has a strong influence on the sampling depth 305 [40]. The sampling depth of a prototype Raman PhAT probe with a $3 \mathrm{~mm}$ diameter laser 306 spot size was reported to be $2 \mathrm{~mm}$ for theophylline discs, using the "infinitely thick" 307 method [36]. However, it has been shown that the sampling depth is less for pressed 308 material compared to uncompacted powders [40]. 
310 unsieved Avicel, the intensity of the aspirin peak at $1606 \mathrm{~cm}^{-1}$ became constant at a depth

311 of $3.5-3.9 \mathrm{~mm}$ for all the mixtures. This indicates that similar to the information depth

312 measurements, the depth at which an infinitely thick sample is achieved is influenced

313 principally by the main component and is less affected by particle size variations of the

314 minor component. If the sampling depth is $3.5 \mathrm{~mm}$ for mixtures of Avicel and aspirin and

315 it is assumed that all layers within the $3.5 \mathrm{~mm}$ contribute equally to the Raman signal,

316 then with a $6 \mathrm{~mm}$ diameter laser spot the sampling volume is $99.0 \mathrm{~mm}^{3}$. This equates to a

317 mass of $0.045 \mathrm{~g}$ if the density (tap) of the powder is assumed to be that of Avicel

$318\left(0.45 \mathrm{~g} \mathrm{~cm}^{-3}\right)$. However, it can be estimated from the information depth plots that

319 approximately $90 \%$ of the Raman signal is generated in the upper $1 \mathrm{~mm}$ layer of the

320 powder; this is consistent with the results of Monte Carlo simulations by Matousek and

321 Parker [24]. Therefore, the mass of sample that contributes to approximately $90 \%$ of the

322 Raman signal is $0.013 \mathrm{~g}$.

323 The depth at which a sample becomes infinitely thick is generally greater than the

324 information depth determined using a reference layer of $\mathrm{TiO}_{2}$. When a reference layer is

325 used, the laser photons propagate through the powder to the reference layer where Raman

326 photons of the reference material are generated. The backscattered Raman photons then

327 propagate back through the powder where they are detected. Consequently, the Raman

328 signal used to determine the information depth is only generated in the plane of the

329 reference layer. In comparison, when a compound peak is used Raman photons can be

330 generated throughout the entire depth of the powder. In such situations, the Raman signal

331 decays slower than the exciting laser intensity $[49,50]$ and so it might be expected that 
332 the sampling depth determined using this method will be greater. As the infinitely thick

333 depth is determined using a signal from the sample powder, this will reflect more

334 accurately the depth of material sampled by the Raman probe during powder blending.

\section{3.4. Effect of particle size on Raman intensities}

337 The second derivative Raman intensity of aspirin, Avicel and sodium nitrate was

338 measured for each particle size range with a powder depth of $8.48 \mathrm{~mm}$, well in excess of

339 the depth required to achieve an infinitely thick sample for each compound (see Figure

340 4). The aspirin peak intensity at $1606 \mathrm{~cm}^{-1}$ increases then decreases with increasing

341 particle size, with the maximum (i.e. largest negative) signal intensity occurring at the

342 intermediate particle size ranges. A similar trend was observed for the sodium nitrate and

343 Avicel peak intensities at 1068 and $1095 \mathrm{~cm}^{-1}$, respectively.

$344 \quad$ Kubelka-Munk theory states that diffuse reflectance increases as particle size

345 decreases, which limits the volume of sample contributing to the Raman signal, and

346 therefore, the Raman intensity should increase with increasing particle size [46, 47]. The

347 Raman signals for aspirin, sodium nitrate and Avicel increase with particle size at lower

348 particle sizes, in accordance with Kubelka-Munk theory; however, the signal decreases

349 with an increase in particle size for larger particles. A similar trend to that observed in

350 Figure 4 was observed in an earlier study by Wang et al. [40], when the probe to sample

351 distance was increased to $10 \mathrm{~mm}$ to increase the unfocused spot diameter illuminating the

352 sample to $\sim 5 \mathrm{~mm}$, and also by Hu et al. [37] when monitoring different particle sizes of

353 flufenamic acid (form I) with small sampling volume probes ( 0.25 and 0.5 inch diameter

354 immersion optics with a $60 \mu \mathrm{m}$ laser spot size). In both of these studies, the maximum 
355 Raman signal intensity was observed for most samples in the intermediate particle size

356 range [37, 40]. Although the variation in intensity with particle size predicted by

357 Kubelka-Munk theory was not observed experimentally in a number of studies [37, 40,

358 51], Wang et al. [40] demonstrated that there was a strong correlation between the Raman

359 intensity and diffuse reflectance. The main reason for the difference is that Kubelka-

360 Munk theory assumes confocal excitation and collection, but in the absence of diffuse

361 reflectance it is likely that the optical configurations employed in the experimental

362 studies had incomplete overlap of the collection and excitation cones. So while an

363 increase in diffuse reflectance decreases the sampling depth, it also increases lateral

364 spreading of the exciting laser beam. This generates additional Raman photons in the

365 region of the collection fibre, which caused an increase in the Raman signal with a

366 decrease in particle size observed in studies by Wang et al. (with a $0.5 \mathrm{~mm}$ laser spot size

367 and a probe to sample distance of $1 \mathrm{~mm}$ ) [40] and by Pellow-Jarman et al. [51]. When the

368 probe to sample distance was increased in the study by Wang et al. [40] (as discussed

369 above), there was greater overlap between the collection and excitation cones and so the

370 improved overlap of the two cones caused by lateral spreading of the excitation beam is

371 less important; hence, the different trends observed at the two different sample to probe

372 distances (and beam diameters).

373 It is apparent that the variation in Raman signal observed with particle size is

374 highly dependent on the optical arrangement employed [40]; it is a balance between

375 signal enhancement through lateral spreading of the beam and signal reduction through a

376 decrease in the sampling depth caused by diffuse reflectance. The PhAT probe mainly

377 used in the present study (6 $\mathrm{mm}$ laser beam diameter), has good overlap of the excitation 
378 and collection cones because of very weak divergence of the beam (i.e. close to a

379 collimated beam with a large depth of field of approximately \pm 1 inch) [52].

380 Consequently, signal enhancement by the lateral beam spreading is less important and so

381 the signal intensity is correlated to the sampling depth (as predicted by Kubelka-Munk

382 theory) up to a certain particle size. For larger particle sizes, it may be that Raman

383 photons are emitted outwith the collection volume of the PhAT probe owing to the 384 greater propagation distances.

\section{3.5. Powder Blending}

387 Different masses and particle sizes of aspirin were added to $75 \mathrm{~g}$ Avicel PH-101 as it was 388 agitated in the mixer. Example second derivative spectra obtained during the mixing of $38940 \mathrm{~g}$ of $250-300 \mu \mathrm{m}$ aspirin particles and Avicel PH-101 are shown in Figure 5; aspirin 390 was added to the Avicel at $120 \mathrm{~s}$. The Raman intensities of the aspirin peaks in the 391 measured spectra increased as the mass of the aspirin was increased and correspondingly

392 the Avicel peak intensity decreased. Mixing profiles were produced by plotting the

393 second derivative Raman intensity against time for the aspirin peaks at 751 and

$3941606 \mathrm{~cm}^{-1}$. The same trends were noted for measurements at each peak so only the results 395 based on $1606 \mathrm{~cm}^{-1}$ are discussed. The mixing profiles for addition of $0-40 \mathrm{~g}$ of the 250 $396-300 \mu \mathrm{m}$ aspirin particles are shown in Figure 6. On addition of aspirin after $120 \mathrm{~s}$, a 397 large increase in Raman scattering occurred at $1606 \mathrm{~cm}^{-1}$ as a result of the mixing 398 properties of the mixer, whereby the aspirin powder is drawn down towards the bottom of 399 the vessel and then up the side of the vessel through the Raman observation zone. As 400 mixing continued, the $2^{\text {nd }}$ derivative signal of aspirin became less negative as the mixture 
401 became homogenous, with the larger masses of aspirin taking longer to achieve a more 402 constant plateau signal.

403 The mixing profiles for the other two particle sizes of aspirin were similar to those

404 in Figure 6, however, the time taken to achieve a homogeneous mixture and the variation 405 (peak-to-peak noise) of the profile at the plateau region increased with particle size for 406 each mass of aspirin added. Bellamy et al. [31] obtained similar mixing profiles to those 407 reported here when non-invasive NIR spectrometry was used to monitor the addition of 408 aspirin to Avicel PH-101 in the same type of mixer. The lesser variability in the Raman 409 profile signal at the plateau region when mixing smaller aspirin particles is due to the 410 more consistent number of aspirin particles passing through the measurement region at 411 any time than occurs for mixing of larger particle sizes; a similar phenomenon was 412 observed by Bellamy et al. [31] for the NIR-based mixing profiles. The magnitude of the 413 mean Raman signal in the plateau region at $700-900 \mathrm{~s}$ was calculated for each of the 414 mixing profiles and plotted against the mass of aspirin added. A linear response curve 415 was generated for each particle size range of aspirin with slopes of $-0.278\left(\mathrm{R}^{2}=\right.$ $4160.986),-0.329\left(R^{2}=0.998\right)$, and $-0.312\left(R^{2}=0.997\right)$ obtained for $<106,250-300$ and $417425-500 \mu \mathrm{m}$ particles, respectively. The lesser sensitivity obtained with the $<106 \mu \mathrm{m}$ 418 particles is probably related to the different shape of these particles (more granular than 419 needle-shaped) identified from microscope images. It is likely that with the large 420 sampling volume used in this study, the change in the relative number of particles with 421 aspirin particle size becomes less significant and so the spectrum obtained is still 422 representative of the mass fraction of aspirin [37]. The estimated sampling volume with a 423 mixing speed of $50 \mathrm{rpm}$ and a sampling depth of $3.5 \mathrm{~mm}$ is $11095 \mathrm{~mm}^{3}$ (assuming all 
424 layers contribute equally), which equates to a mass of $4.99 \mathrm{~g}$. However, if $90 \%$ of the

425 Raman signal is generated in the top $1 \mathrm{~mm}$ layer of powder, then most of the measured 426 signal arises from $1.43 \mathrm{~g}$ of sample.

427 The NIR method of Bellamy et al. [31] and a previously reported procedure for 428 monitoring powder blending by broadband acoustic emission spectrometry [41] were 429 used simultaneously with Raman spectrometry to compare the mixing profiles obtained 430 by the three non-invasive techniques. Example profiles are given in Figure 7 for addition 431 of $30 \mathrm{~g}$ aspirin to $75 \mathrm{~g}$ Avicel $\mathrm{PH}-101$ mixing at $50 \mathrm{rpm}$. The features of the mixing 432 profiles, including the end-point of blending to a homogeneous mixture, were similar for 433 the three techniques, although the signal to noise was best for NIR and poorest for 434 acoustic emission. De Beer et al. [25] also found in-line Raman and NIR spectrometry 435 produced similar end-points (defined by the content uniformity method [30]) when used 436 simultaneously to monitor mixing of Avicel PH-102, lactose DCL 21 and silicium 437 dioxide in a high shear blender. However, there is clearer correspondence between the 438 mixing profiles over the entire blending experiment in the present study, as the mixing 439 profiles were derived from the spectral signals related to the added component and the 440 spectral measurements were acquired at a higher acquisition frequency. Table 1 gives a 441 comparison of the detection limit for unsieved aspirin mixing at $50 \mathrm{rpm}$ in unsieved 442 Avicel PH101 for non-invasive NIR spectrometry, Raman spectrometry, and acoustic 443 emission. The detection limit calculated for Raman measurements was about an order of 444 magnitude poorer than that obtained with NIR spectrometry with a Zeiss Corona 445 instrument, but 5-fold better than the value obtained for acoustic emission with a Nano30 446 transducer. 
448 and then $25 \mathrm{~g}$ aspartame (after $900 \mathrm{~s}$ ) to $75 \mathrm{~g}$ of Avicel PH-101. Inspection of the second

449 derivative spectra of the compounds revealed that mixing profiles could be plotted based

450 on well resolved peaks for aspirin or aspartame with minimal interference from the other

451 compounds; this is an advantage over NIR spectrometry for monitoring multiple

452 component systems where multivariate techniques are generally needed to deconvolute

453 the relative contributions from each compound. Example Raman mixing profiles based

454 on the aspirin peak at $1606 \mathrm{~cm}^{-1}$ and the aspartame peak at $1006 \mathrm{~cm}^{-1}$ are shown in Figure

455 8. As observed in Figure 6, on addition of aspirin to the mixer at $120 \mathrm{~s}$, there was an

456 initial large increase in the aspirin signal, which then decreased to a constant value; as

457 expected, the aspartame response remained at approximately zero when aspirin was

458 added to the Avicel. However, on addition of aspartame to the vessel at $900 \mathrm{~s}$, there was a

459 large change in the aspartame signal at about $1000 \mathrm{~s}$. The oscillating mixing profile of

460 aspartame between 1200 and $1600 \mathrm{~s}$ is characteristic of a cohesive particle [32]. The

461 aspirin signal was affected by the addition of aspartame, initially increasing slightly

462 owing to minor compaction of the powder mixture in the vessel followed by a reduction

463 owing to dilution of the aspirin concentration as aspartame is blended in to the mixture.

\section{4. Conclusions}

466 This study has shown that non-invasive Raman spectrometry with an optically broadened

467 spot size of $6 \mathrm{~mm}$ gives sampling depths of over $3 \mathrm{~mm}$ for measurement of aspirin in

468 Avicel; this is greater than the value obtained for non-invasive NIR spectrometry in a 469 previous study [31]. In situ Raman measurements can be used to produce mixing profiles 
470 to study how the powders mix together, identify when the end point of mixing has

471 occurred, and perform quantitative analysis in real time. Although less sensitive than a 472 previously reported non-invasive NIR procedure, the Raman method could offer 473 advantages when optimising mixing regimes for multi-component samples, as it may 474 allow individual compound specific mixing profiles to be produced because of the 475 technique's greater chemical specificity.

\section{Acknowledgements}

478 The support of EPSRC/DTI through LINK grant GR/R/19366/01 is acknowledged.

479 CPACT is thanked for funding PA and LJB's PhD studentships, and the Royal Society is 480 thanked for the award of a University Research Fellowship to AN. 


\section{References}

484 [1] J. Rantanen, Process analytical applications of Raman spectroscopy, J. Pharm. 485 Pharmacol., 59 (2007) 171-177.

486 [2] T. De Beer, A. Burggraeve, M. Fonteyne, L. Saerens, J.P. Remon, C. Vervaet, Near 487 infrared and Raman spectroscopy for the in-process monitoring of pharmaceutical 488 production processes, Int. J. Pharm., 417 (2011) 32-47.

489 [3] M. Dyrby, S.B. Engelsen, L. Nørgaard, M. Bruhn, L. Lundsberg-Nielsen, 490 Chemometric quantitation of the active substance (containing $\mathrm{C} \mathrm{N}$ ) in a pharmaceutical 491 tablet using near-infrared (NIR) transmittance and NIR FT-Raman spectra, Appl. 492 Spectrosc., 56 (2002) 579-585.

493 [4] C.G. Kontoyannis, Quantitative determination of $\mathrm{CaCO}_{3}$ and glycine in antacid tablets 494 by laser Raman spectroscopy, J. Pharm. Biomed. Anal., 13 (1995) 73-76.

495 [5] R. Szostak, S. Mazurek, FT-Raman quantitative determination of ambroxol in tablets, 496 J. Mol. Struct., 704 (2004) 229-233.

497 [6] R. Szostak, S. Mazurek, Quantitative determination of acetylsalicylic acid and 498 acetaminophen in tablets by FT-Raman spectroscopy, Analyst, 127 (2002) 144-148.

499 [7] L.S. Taylor, F.W. Langkilde, Evaluation of solid-state forms present in tablets by 500 Raman spectroscopy, J. Pharm. Sci., 89 (2000) 1342-1353. 
501 [8] G.J. Vergote, C. Vervaet, J.P. Remon, T. Haemers, F. Verpoort, Near-infrared FT-

502 Raman spectroscopy as a rapid analytical tool for the determination of diltiazem

503 hydrochloride in tablets, Eur. J. Pharm. Sci., 16 (2002) 63-67.

504 [9] C. Wang, T.J. Vickers, C.K. Mann, Direct assay and shelf-life monitoring of aspirin 505 tablets using Raman spectroscopy, J. Pharm. Biomed. Anal., 16 (1997) 87-94.

506 [10] H. Wikström, S. Romero-Torres, S. Wongweragiat, J.A.S. Williams, E.R. Grant,

507 L.S. Taylor, On-line content uniformity determination of tablets using low-resolution

508 Raman spectroscopy, Appl. Spectrosc., 60 (2006) 672-681.

509 [11] S. Mazurek, R. Szostak, Quantitative determination of diclofenac sodium in solid

510 dosage forms by FT-Raman spectroscopy, J. Pharm. Biomed. Anal., 48 (2008) 814-821.

511 [12] S.E.J. Bell, D.T. Burns, A.C. Dennis, L.J. Matchett, J.S. Speers, Composition

512 profiling of seized ecstasy tablets by Raman spectroscopy, Analyst, 125 (2000) 18115131815.

514 [13] S. Mazurek, R. Szostak, Quantitative determination of captopril and prednisolone in 515 tablets by FT-Raman spectroscopy, J. Pharm. Biomed. Anal., 40 (2006) 1225-1230.

516 [14] M.G. Orkoula, C.G. Kontoyannis, Non-destructive quantitative analysis of 517 risperidone in film-coated tablets, J. Pharm. Biomed. Anal., 47 (2008) 631-635.

518 [15] M. Kim, H. Chung, Y. Woo, M. Kemper, New reliable Raman collection system 519 using the wide area illumination (WAI) scheme combined with the synchronous intensity 
520 correction standard for the analysis of pharmaceutical tablets, Anal. Chim. Acta, 579

521 (2006) 209-216.

522 [16] J. Johansson, A. Sparén, O. Svensson, S. Folestad, M. Claybourn, Quantitative

523 transmission Raman spectroscopy of pharmaceutical tablets and capsules, Appl.

524 Spectrosc., 61 (2007) 1211-1218.

525 [17] M. Fransson, J. Johansson, A. Sparén, O. Svensson, Comparison of multivariate

526 methods for quantitative determination with transmission Raman spectroscopy in

527 pharmaceutical formulations, J. Chemom., 24 (2010) 674-680.

528 [18] A. Sparén, J. Johansson, O. Svensson, S. Folestad, M. Claybourn, Transmission

529 Raman spectroscopy for quantitative analysis of pharmaceutical solids, Am. Pharm. Rev., $530 \quad 12(2009) 62-71$.

531 [19] J. Kim, J. Noh, H. Chung, Y.-A. Woo, M.S. Kemper, Y. Lee, Direct, non-destructive 532 quantitative measurement of an active pharmaceutical ingredient in an intact capsule 533 formulation using Raman spectroscopy, Anal. Chim. Acta, 598 (2007) 280-285.

534 [20] C. Eliasson, N.A. Macleod, L.C. Jayes, F.C. Clarke, S.V. Hammond, M.R. Smith, P. 535 Matousek, Non-invasive quantitative assessment of the content of pharmaceutical 536 capsules using transmission Raman spectroscopy, J. Pharm. Biomed. Anal., 47 (2008) $537 \quad 221-229$.

538 [21] P. Matousek, A.W. Parker, Non-invasive probing of pharmaceutical capsules using 539 transmission Raman spectroscopy, J. Raman Spectrosc., 38 (2007) 563-567. 
540 [22] K. Buckley, P. Matousek, Recent advances in the application of transmission Raman

541 spectroscopy to pharmaceutical analysis, J. Pharm. Biomed. Anal., 55 (2011) 645-652.

542 [23] M.D. Hargreaves, N.A. Macleod, M.R. Smith, D. Andrews, S.V. Hammond, P.

543 Matousek, Characterisation of transmission Raman spectroscopy for rapid quantitative 544 analysis of intact multi-component pharmaceutical capsules, J. Pharm. Biomed. Anal., 54 545 (2011) 463-468.

546 [24] P. Matousek, A.W. Parker, Bulk Raman analysis of pharmaceutical tablets, Appl. 547 Spectrosc., 60 (2006) 1353-1357.

548 [25] T.R.M. De Beer, C. Bodson, B. Dejaegher, B. Walczak, P. Vercruysse, A. 549 Burggraeve, A. Lemos, L. Delattre, Y.V. Heyden, J.P. Remon, C. Vervaete, W.R.G. 550 Baeyens, Raman spectroscopy as a process analytical technology (PAT) tool for the in551 line monitoring and understanding of a powder blending process, J. Pharm. Biomed. 552 Anal., 48 (2008) 772-779.

553 [26] G.J. Vergote, T.R.M. De Beer, C. Vervaet, J.P. Remon, W.R.G. Baeyens, N. 554 Diericx, F. Verpoort, In-line monitoring of a pharmaceutical blending process using FT555 Raman spectroscopy, Eur. J. Pharm. Sci., 21 (2004) 479-485.

556 [27] D.S. Hausman, R.T. Cambron, A. Sakr, Application of Raman spectroscopy for on557 line monitoring of low dose blend uniformity, Int. J. Pharm., 298 (2005) 80-90.

558 [28] S.S. Sekulic, H.W. Ward, D.R. Brannegan, E.D. Stanley, C.L. Evans, S.T. 559 Sciavolino, P.A. Hailey, P.K. Aldridge, On-line monitoring of powder blend 560 homogeneity by near-infrared spectroscopy, Anal. Chem., 68 (1996) 509-513. 
561 [29] O. Berntsson, L.G. Danielsson, B. Lagerholm, S. Folestad, Quantitative in-line 562 monitoring of powder blending by near infrared reflection spectroscopy, Powder 563 Technol., 123 (2002) 185-193.

564 [30] C. Bodson, W. Dewe, P. Hubert, L. Delattre, Comparison of FT-NIR transmission 565 and UV-vis spectrophotometry to follow the mixing kinetics and to assay low-dose 566 tablets containing riboflavin, J. Pharm. Biomed. Anal., 41 (2006) 783-790.

567 [31] L.J. Bellamy, D. Littlejohn, A. Nordon, Real-time monitoring of powder mixing in a 568 convective blender using non-invasive reflectance NIR spectrometry, Analyst, 133 569 (2008) 58-64.

570 [32] L.J. Bellamy, A. Nordon, D. Littlejohn, Effects of particle size and cohesive 571 properties on mixing studied by non-contact NIR, Int. J. Pharm., 361 (2008) 87-91.

572 [33] D. Ely, S. Chamarthy, M.T. Carvajal, An investigation into low dose blend 573 uniformity and segregation determination using NIR spectroscopy, Colloid Surf. A574 Physicochem. Eng. Asp., 288 (2006) 71-76.

575 [34] J. Johansson, S. Pettersson, S. Folestad, Characterization of different laser irradiation 576 methods for quantitative Raman tablet assessment, J. Pharm. Biomed. Anal., 39 (2005) $577 \quad 510-516$.

578 [35] Y. Xie, W. Tao, H. Morrison, R. Chiu, J. Jona, J. Fang, N. Cauchon, Quantitative 579 determination of solid-state forms of a pharmaceutical development compound in drug 580 substance and tablets, Int. J. Pharm., 362 (2008) 29-36. 
581 [36] H. Wikström, I.R. Lewis, L.S. Taylor, Comparison of sampling techniques for in-

582 line monitoring using Raman spectroscopy, Appl. Spectrosc., 59 (2005) 934-941.

583 [37] Y. Hu, H. Wikström, S.R. Byrn, L.S. Taylor, Analysis of the effect of particle size

584 on polymorphic quantitation by Raman spectroscopy, Appl. Spectrosc., 60 (2006) 977585984.

586 [38] M.V. Schulmerich, W.F. Finney, R.A. Fredricks, M.D. Morris, Subsurface Raman 587 spectroscopy and mapping using a globally illuminated non-confocal fiber-optic array 588 probe in the presence of Raman photon migration, Appl. Spectrosc., 60 (2006) 109-114.

589 [39] J. Rantanen, H. Wikström, F.E. Rhea, L.S. Taylor, Improved understanding of 590 factors contributing to quantification of anhydrate/hydrate powder mixtures, Appl. 591 Spectrosc., 59 (2005) 942-951.

592 [40] H.L. Wang, C.K. Mann, T.J. Vickers, Effect of powder properties on the intensity of 593 Raman scattering by crystalline solids, Appl. Spectrosc., 56 (2002) 1538-1544.

594 [41] P. Allan, L.J. Bellamy, A. Nordon, D. Littlejohn, Non-invasive monitoring of the 595 mixing of pharmaceutical powders by broadband acoustic emission, Analyst, 135 (2010) $596 \quad 518-524$

597 [42] R.C. Rowe, P.J. Sheskey, P.J. Weller, Handbook of Pharmaceutical Excipients, 4th 598 ed., Pharmaceutical Press, London, 2003. 
599 [43] F.C. Clarke, S.V. Hammond, R.D. Jee, A.C. Moffat, Determination of the 600 information depth and sample size for the analysis of pharmaceutical materials using 601 reflectance near-infrared microscopy, Appl. Spectrosc., 56 (2002) 1475-1483.

602 [44] O. Berntsson, L.G. Danielsson, S. Folestad, Estimation of effective sample size 603 when analysing powders with diffuse reflectance near-infrared spectrometry, Anal. Chim. 604 Acta, 364 (1998) 243-251.

605 [45] U. Balachandran, N.G. Eror, Raman spectra of titanium dioxide, J. Solid State 606 Chem., 42 (1982) 276-282.

607 [46] B. Schrader, G. Bergmann, Die intensität des Ramanspektrums polykristalliner 608 substanzen I. Strahlingsbilanz von substanz und probenanordnung, Z. Anal. Chem. Fres., $609225(1967) 230-247$.

610 [47] B. Schrader, A. Hoffmann, S. Keller, Near-infrared Fourier-transform Raman 611 spectroscopy - facing absorption and background, Spectrochim. Acta, 47A (1991) 11356121148.

613 [48] O. Berntsson, T. Burger, S. Folestad, L.G. Danielsson, J. Kuhn, J. Fricke, Effective 614 sample size in diffuse reflectance near-IR spectrometry, Anal. Chem., 71 (1999) 617-623.

615 [49] N. Everall, T. Hahn, P. Matousek, A.W. Parker, M. Towrie, Picosecond time616 resolved Raman spectroscopy of solids: Capabilities and limitations for fluorescence 617 rejection and the influence of diffuse reflectance, Appl. Spectrosc., 55 (2001) 1701-1708. 
618 [50] N. Everall, T. Hahn, P. Matousek, A.W. Parker, M. Towrie, Photon migration in

619 Raman spectroscopy, Appl. Spectrosc., 58 (2004) 591-597.

620 [51] M.V. Pellow-Jarman, P.J. Hendra, R.J. Lehnert, The dependence of Raman signal

621 intensity on particle size for crystal powders, Vib. Spectrosc., 12 (1996) 257-261.

622 [52] H. Owen, D.J. Strachan, J.B. Slater, J.M. Tedesco, Large-collection-area optical 623 probe, US Pat., WO2005060622 A2, 2005.

624

625

626 


\section{Table captions}

628 Table 1. Detection limit for aspirin in Avicel PH-101 mixing at $50 \mathrm{rpm}$ for non-invasive

629 NIR spectrometry, acoustic emission (AE) and Raman spectrometry.

630

\begin{tabular}{ccc}
\hline Technique & Signal & Detection limit $/(\% \mathrm{w} / \mathrm{w})^{\mathrm{a}}$ \\
\hline NIR & $1^{\text {st }}$ derivative of $\log (1 / \mathrm{R})$ at $8956 \mathrm{~cm}^{-1}$ & 0.1 \\
Raman & $2^{\text {nd }}$ derivative at $1606 \mathrm{~cm}^{-1}$ & 1.1 \\
$\mathrm{AE}$ & Area between 0 and $400 \mathrm{kHz}$ & 5.2 \\
\hline
\end{tabular}

631

$632{ }^{a}$ Detection limit calculated from 3 times the standard deviation of the signal between 702

633 and $900 \mathrm{~s}$ in the mixing profile for Avicel PH-101 alone $(\mathrm{n}=34)$ divided by the

634 sensitivity of the signal response for aspirin. The sensitivity was obtained from a plot of

635 concentration $(0-28.6 \% \mathrm{w} / \mathrm{w}$ for $\mathrm{AE}$ and $0-34.8 \% \mathrm{w} / \mathrm{w}$ for NIR and Raman) against

636 average signal intensity between 702 and $900 \mathrm{~s}$ in the mixing profiles $(\mathrm{n}=34)$. The NIR

637 and Raman data were resampled to match the acquisition frequency of the AE

638

measurements (every $6 \mathrm{~s}$ ).

639

640 


\section{Figure captions}

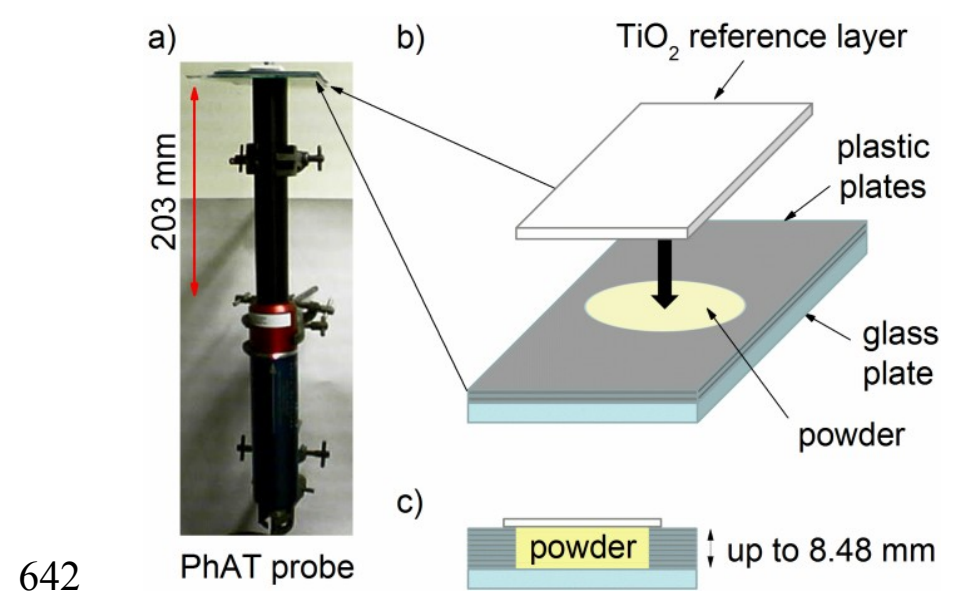

643 Figure 1. a) Raman PhAT probe set up for sampling depth experiments with powders, b)

644 expansion of sample set up showing the glass plate, plastic plates and $\mathrm{TiO}_{2}$ reference 645 layer, and c) end on view of sample set up. 


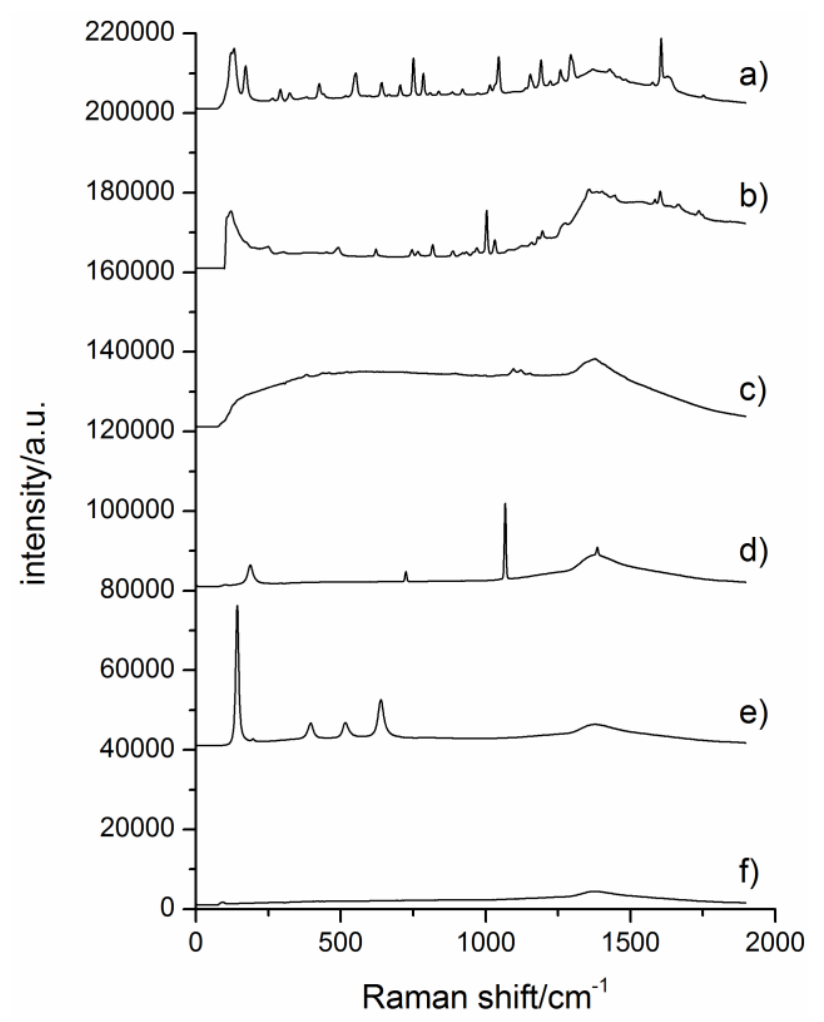

646

647 Figure 2. Offset Raman spectra of a) aspirin, b) aspartame, c) Avicel PH-101, d) sodium

648 nitrate, e) $\mathrm{TiO}_{2}$ reference layer (intensity reduced by a factor of 4) and f) $2.68 \mathrm{~mm}$ thick

649 glass plate. All spectra, except for aspartame, were acquired using the PhAT probe with

650 an exposure time of $0.5 \mathrm{~s}$ and 1 accumulation. The spectrum for aspartame was acquired

651 using a MR probe with an exposure time of $4.0 \mathrm{~s}$ and 1 accumulation.

652 


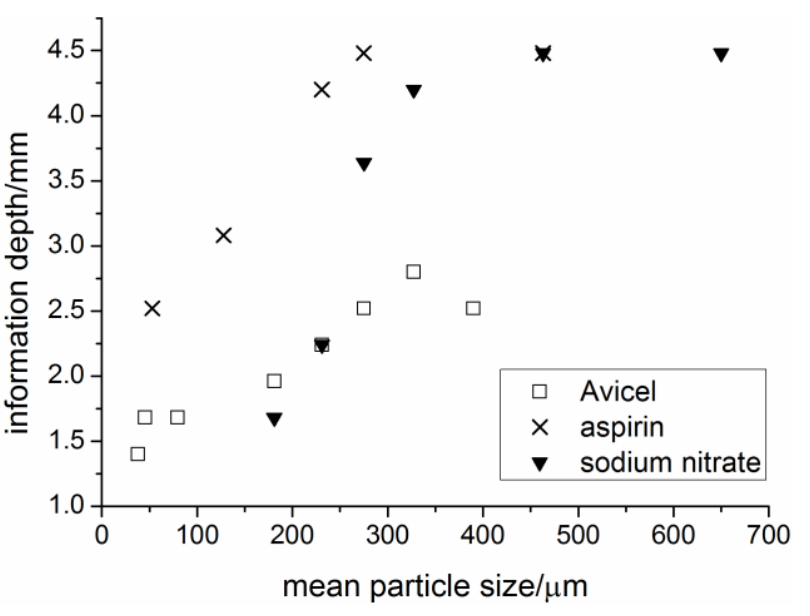

654 Figure 3. Information depths of Avicel, aspirin and sodium nitrate of different particle 655 sizes, based on measurements of the $\mathrm{TiO}_{2}$ peak at $397 \mathrm{~cm}^{-1}$. 


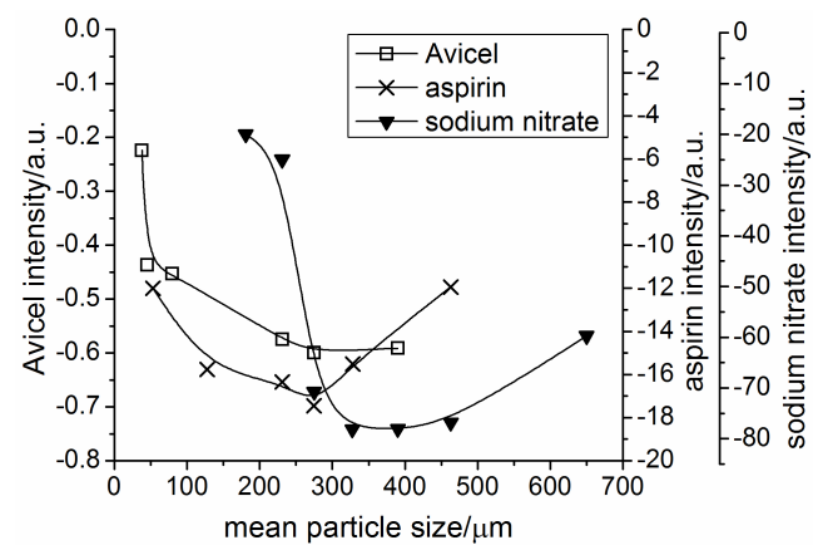

658 Figure 4. Second derivative Raman signal intensity for measurements at aspirin $659\left(1606 \mathrm{~cm}^{-1}\right)$, Avicel $\left(1095 \mathrm{~cm}^{-1}\right)$ and sodium nitrate $\left(1068 \mathrm{~cm}^{-1}\right)$ peaks for increasing 660 particle size ranges with a powder depth of $8.48 \mathrm{~mm}$. The lines drawn through the data 661 points were calculated using a cubic B-spline function. 


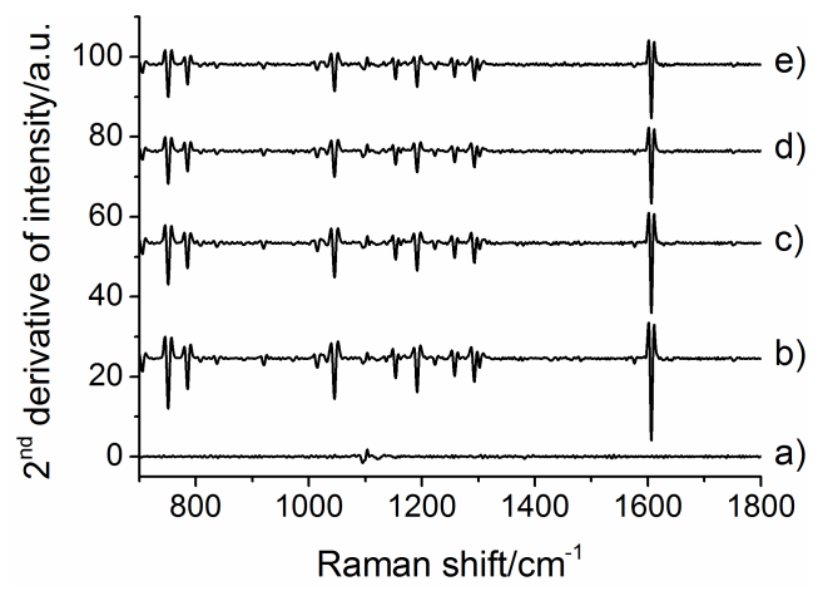

664 Figure 5. Offset second derivative Raman spectra obtained at a) 50, b) 150, c) 200, d) 400

665 and e) $800 \mathrm{~s}$ during the mixing of $40 \mathrm{~g}$ of aspirin (particle size range $250-300 \mu \mathrm{m}$ ) and

666 Avicel PH-101. Aspirin was added to Avicel at $120 \mathrm{~s}$ and a mixing speed of $50 \mathrm{rpm}$ was 667 used.

668 


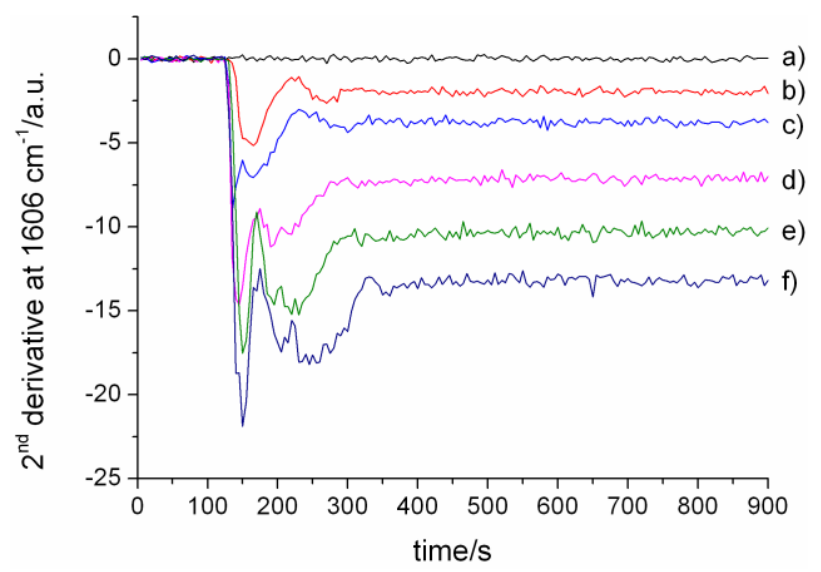

670 Figure 6. Raman PhAT probe mixing profiles at $1606 \mathrm{~cm}^{-1}$ for additions of a) 0 , b) 5 , c)

67110 , d) 20, e) 30 and f) $40 \mathrm{~g}$ of aspirin (particle size range $250-300 \mu \mathrm{m}$ ) to Avicel PH-

672101 at $120 \mathrm{~s}$, mixing with an impeller speed of $50 \mathrm{rpm}$.

673 


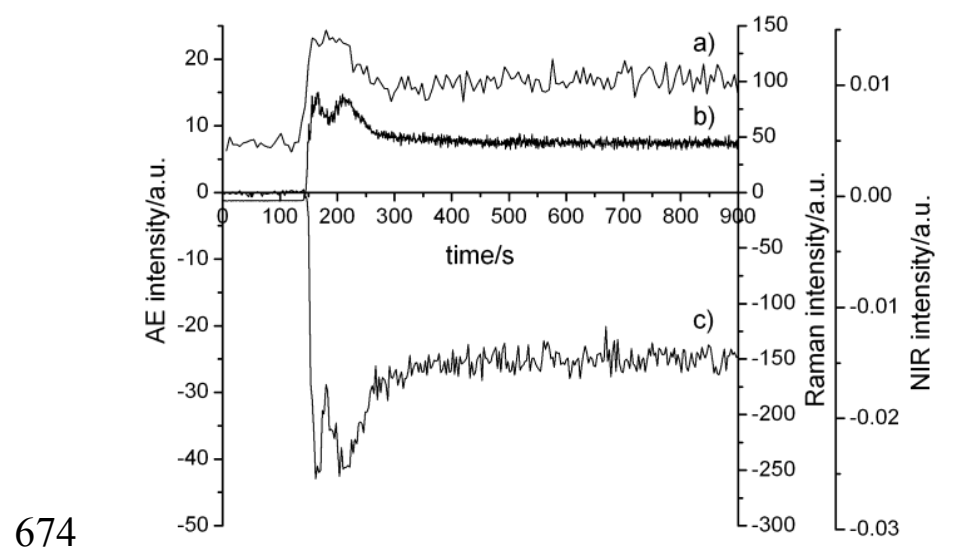

675 Figure 7. Mixing profiles for addition of $30 \mathrm{~g}$ aspirin to $75 \mathrm{~g}$ Avicel PH-101 at $120 \mathrm{~s}$ 676 mixing at $50 \mathrm{rpm}$, obtained simultaneously by three non-invasive techniques: a) acoustic 677 emission spectrometry (area between 0 and $400 \mathrm{kHz}$ ); b) NIR spectrometry ( $1^{\text {st }}$ derivative 678 of $\log (1 / \mathrm{R})$ at $\left.8956 \mathrm{~cm}^{-1}\right)$, and c) Raman spectrometry $\left(2^{\text {nd }}\right.$ derivative of intensity at $\left.6791606 \mathrm{~cm}^{-1}\right)$.

680 


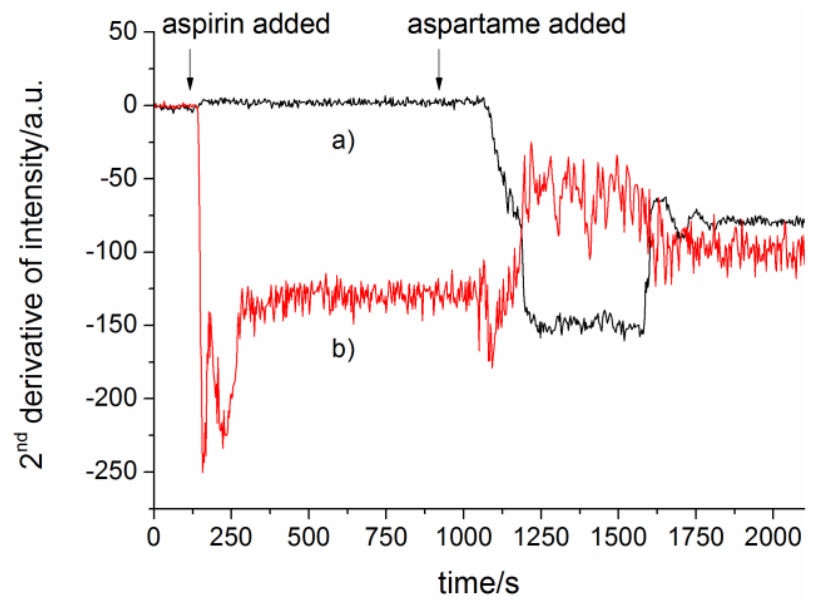

682 Figure 8. Raman mixing profiles obtained using a) the aspartame peak at $1006 \mathrm{~cm}^{-1}$ and $683 \mathrm{~b}$ ) the aspirin peak at $1606 \mathrm{~cm}^{-1}$ for addition of $25 \mathrm{~g}$ aspirin $(120 \mathrm{~s})$ and $25 \mathrm{~g}$ aspartame 684 (900 s) to $75 \mathrm{~g}$ Avicel PH-101 mixing at $50 \mathrm{rpm}$. 